\title{
Obesity in postmenopausal women: causes, prevalence and specific risks: role of decreased resting energy expenditure
}

\section{Causes and differential consequences of overweight and obesity in women}

All epidemiological studies relating mortality to Body Mass Index (BMI) in adults up to 80 years show the typical U-curve, with the minimum mortality around BMI of 22 in both sexes, an abrupt increase in mortality when BMI is below 18 , and a steady increase in mortality when BMI exceeds 28. According to a major prospective study involving 900000 adults, every $5 \mathrm{~kg} / \mathrm{m}^{2}$ increase in BMI over $25 \mathrm{~kg} / \mathrm{m} 2$ increases mortality by $30 \% .{ }^{1}$ Despite the OMS's definition as a disease, the large heterogeneity, of the "Obesity" deserves the qualification of a syndrome or "set of diseases" with different causes, pathophysiology and prognosis, but whose common feature is the excessive storage of fat. ${ }^{2}$

Epidemiological data worldwide show the higher prevalence of obesity in women, and the trend seems to be increasing. ${ }^{3}$ Many sociological and cultural factors have an influence, but undoubtedly the role played by hormonal changes and emotional and psychological factors in women are enormously important, and they are often interrelated. Polycystic ovarian disease, thyroid disease, which are more prevalent in women, and the periods of adolescence, pregnancy and menopause are examples of situations that make susceptible women much more vulnerable to fat storage.

In a cross-sectional study of 900 cases of patients with obesity grades 2 and 3, we showed that the time of onset of weight gain in women was fundamentally related to pregnancy, menopause and emotional situations, while in men smoking cessation and a decrease in physical sports activity predominated. ${ }^{4}$ Likewise, a study of psychological affectation and multiscale quality of life in the same population showed greater overall affectation and of any subscale in obese women compared to men. ${ }^{5}$
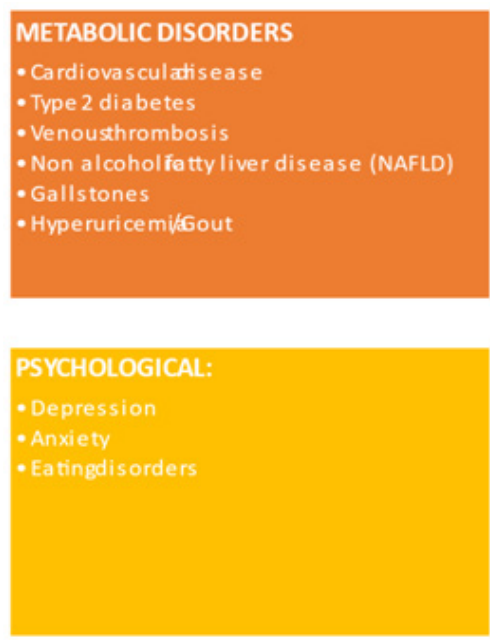

Volume II Issue 5 - 202 I

Clotilde Vázquez, ${ }^{1-3}$ Bogdana L Luca,' Jersy Cárdenas, ${ }^{1,2}$ Alvaro Sanchez, ${ }^{1,4}$ Teresa Montoya, ${ }^{2}$ Paula Labeira, ${ }^{1,4}$ Belen Gutierrez, ${ }^{1,4}$ Yvonne Fernandez,' Roberto Sierra, ${ }^{1,3}$ Diego Meneses'

'Department of Endocrinology and Nutrition, Fundación Jiménez Díaz University Hospital, Spain ${ }^{2}$ Infanta Elena University Hospital, Spain ${ }^{3}$ Villalba General University Hospital, Spain ${ }^{4}$ Medicadiet, Spain

\section{Correspondence: Clotilde Vázquez, Department of Endocrinology and Nutrition. Overweight and Obesity Unit. Fundación Jiménez Díaz University Hospital, Spain, Email cvazquezma@gmail.com}

Received: September 20, 202I | Published: October 05, 2021

As in the case of most heterogeneous diseases, the etiology is obviously multifactorial, where genetic predisposition and the environment, through epigenetic changes and other mechanisms, can favor its appearance. Hormonal variations, emotional factors, concomitant medications, lifestyle and a long etcetera operate here to give rise to a specific clinical phenotypic outcome with its risks and comorbidities. Thus an etiological identification is important to establish a comprehensive individualized treatment (Figure 1).
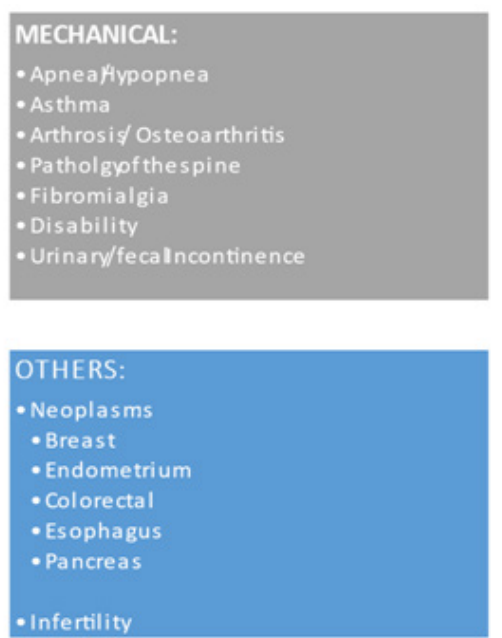

Figure I Obesity-related chronic concomitant diseases in women. 
Leaving aside the dangers and consequences of obesity in pregnancy, many studies have shown that women have higher risks of diabetes, cancer and cardiovascular disease than men for the same BMI range. An important clinical guideline supported by data shows the greater and earlier increase (four times more) in the risk of developing diabetes in women when their BMI is modified between two and three points, by comparing two important cohort studies: the Nurse's Health Study (NHS) and the Health Professionals Follow-up Study (HPS). ${ }^{6}$

The same Nurse's Health Study showed that in women aged 33 to 55 years who were non-smokers and followed for 18 years, the risk of coronary heart disease increased 6-fold from a BMI of 20 to 32 . Moreover, the overall risk of death, specific death from cardiovascular causes, cancer and other causes, increased in this same cohort from a BMI of $25 .{ }^{7}$ Equally striking are the studies of breast cancer survival in women with obesity and diabetes compared to women without these diseases. ${ }^{8}$

On the other hand, obesity is associated with several musculoskeletal conditions that promote disability: osteoarthritis, low back pain, diffuse idiopathic skeletal hyperostosis, gait disturbances, soft tissue disorders, osteoporosis, gout, fibromyalgia and connective tissue disorders. Overweight and obesity are associated in some studies with early and prolonged periods of disability. ${ }^{9}$ The more frequent disability among women relative to men is probably due to the longer survival of women on the one hand, but also because men are physically stronger than women even at older ages and are therefore more likely to recover from mild disability. The finding that overweight and obesity have a more pronounced effect on recovery from disability among women may indicate that excess body weight coupled with lower muscle mass is the more dangerous phenotype.

Although there is a mean difference of 5 years in life expectancy in favor of women, the same difference is not observed when focusing on disease-free life expectancy. Together with the observation of a sex interaction with BMI and waist circumference in the analysis of disability recovery, there is an indication that obese women are more likely to veer to a disabled state.

\section{Obesity and menopause}

It is believed that, of life's several phases, the climacteric is the one that causes the greatest impact on the woman's health. In several epidemiological studies abdominal Obesity is present in two thirds of the women. ${ }^{10}$

At the start of the menopausal transition (MT), the rate of fat gain doubled, and lean mass declined; gains and losses continued until 2 years after as the SWAN study demonstrated. ${ }^{11}$ Their joint rates of change result in no detectable acceleration in weight or BMI at the onset of the MT. That an MT-related acceleration in weight or BMI is not observed, despite the high-velocity increase in fat mass, is concordant with the growing appreciation that, while BMI is a wellestablished, strong composite indicator of cardiometabolic risk, it is a less strong index of adiposity and particular aspects of adiposity such as the location of fat. ${ }^{12}$

Although at the epidemiological level the diagnosis and classification of obesity is still based on BMI, which is an index of corpulence, in clinical practice it is very important to measure the amount of fat, and its location, to better classify the type of obesity, quantify the risks, establish therapeutic targets and an adequate follow up. As a result, BMI is a less useful indicator of cardiometabolic risk in postmenopausal women. That is why it is so important in menopausal women to perform a body composition study, and waist circumference (WC) measurement. WC greater than $88 \mathrm{~cm}$ in women are associated with increased risk of various metabolic and cardiovascular diseases, ${ }^{13}$ while an increase of only $1 \mathrm{~cm}$ in waist circumference is associated with a $2 \%$ increase in the risk of cardiovascular disease. ${ }^{10}$

There are many risk factors for developing obesity in menopause 12 ranging from hormonal acute changes: , especially the decrease in estrogen production, the occurrence of frequent deseases: autoinumne hypothiroidism, hypercholesterolemia, osteoarticular pain and aging itself, and passing by psychological issues such as depressive symptoms, mood swings, irritability with associated emotional intake. Other Lifestyle related errors are identified in the peri menopause period related to the consumption of fast food, processed and refined foods, fiber intake deficit, and a greater sedentary lifestyle. Incorrect and very restrictive diets, skipping meals ... as well as vitamin D deficiency. Sleeping problems associated with hot flashes, which are so frequent during this period, contribute to the tendency to gain weight.

Most of these events are triggered by the decrease in estrogen and its action on thermogenesis, cardiovascular and musculoskeletal system, mood, sleep among others. ${ }^{14}$ In addition, estrogens regulate the levels of hormones and neurotransmitters related to appetite. Menopausal women therefore experience more brain signals of hunger, leading to higher caloric intake and weight gain. ${ }^{15}$

It is well known that low estrogens levels, due to ovarian insufficiency, in conditions such as menopause or ovariectomy are associated with decreased energy expenditure, leading to weight gain and obesity at long term. Concerning energy expenditure, the main effect of estradiol is on brown adipose tissue thermogenesis. ${ }^{14}$

In an cross sectional observational study we demonstrated that patients with obesity have a lower resting energy expenditure (REE) measured by indirect calorimetry than REE calculated by predictive formulas., the difference being greater in women. ${ }^{16}$

It is well known that alterations of the gut microbiota is related to overweight and metabolic disorders. ${ }^{17}$ Besides, low levels of circulating estrogen observed in post-menopausal women can adversely impact gut health. The gut microbiome is one of the principal regulators of circulating estrogens. through secretion of $\beta$-glucuronidase, an enzyme that deconjugates estrogens into their active forms. When this process is impaired through dysbiosis (characterized by poor microbial diversity), the decrease in deconjugation results in a further reduction of circulating estrogens, that contribute to the development of obesity, metabolic syndrome, cancer, endometrial hyperplasia, endometriosis, cardiovascular disease (CVD) and cognitive function. ${ }^{18}$

We performed recently a cross sectional observational study comparing the REE in 126 overweighted postmenopausal women versus 39 adult overweighted women under 50 years. We found a decrease in REE in postmenopausal women. REE was positively associated with weight, BMI, muscle mass and negatively associated with the presence of cardiovascular disease and clinical suspicion of dysbiosis. ${ }^{19}$ Gut disbiosis was suspected when the women complained for symptons affecting the gastrointestinal tract : symptoms such as bloating, nausea, digestive distress, diarrhea, constipation or their alternation, and scored pathologically on the short GIQLi test.

All these factors influencing menopause require a comprehensive approach to this transitional phase in women. ${ }^{20}$ 


\section{Conclusions}

The prevalence of Obesity is increasing worldwide. Epidemiological data show a higher prevalence of obesity in women, and the trend seems to be increasing. The differential consequences are related to gestational pathology, infertility, but also to the higher risk of cardiovascular disease, incidence of diabetes, disability and cancer after menopause.

Pre and menopause is associated with gaining fat and lots of variable amounts of lean mass. This fact causes a great impact on the woman's health. In several epidemiological studies abdominal Obesity is present in two thirds of the postmenopausal women. The abrupt decrease in estrogen secretion and imbalance with androgens causes decreased thermogenesis, increased appetite and other changes related to energy homeostasis. In our experience, the resting energy expenditure of menopausal women decreases considerably, suggesting the need to individualize their treatment, focusing in fat gain, lean losses and actual energy needs.

Given the differential consequences of fat gain in women after menopause and the risks involved. It is important to reset the approach to obesity in women as a serious, chronic and life-threatening disease. In summary, women's overweight and obesity, should no longer be considered as an aesthetic problem, but as a serious health issue, which worsens in vulnerable hormonal periods and after menopause. A nutritional and pharmacological treatment if necessary should be implemented to counteract abdominal fat gain and attenuate metabolic and cardiovascular risk. Early action to prevent or minimize weight gain inmenopausal women is therefore more than justified.

\section{Acknowledgments}

None.

\section{Conflicts of interest}

We disclose no conflicts of interest in this study.

\section{Funding}

None.

\section{References}

1. Prospective Studies Collaboration. Body-mass index and cause-specific mortality in 900000 adults: collaborative analyses of 57 prospective studies. Lancet. 2009;373:1083-1096.

2. Vázquez Martínez Clotilde, Cos Blanco. Ana Isabel de. Manual de Obesidad. $1^{a}$ Ed. Madrid, España:Dykinson; 2019

3. Peters SAE, Muntner P, Woodward M. Sex Differences in the Prevalence of, and Trends in, Cardiovascular Risk Factors, Treatment, and Control in the United States, 2001 to 2016. Circulation. 2019;139:1025-1035.

4. Vázquez C, Alcaraz F, Botella-Carretero JI, et al. Probesci strategy:a cheaper therapeutic approach for obese patients. Nutr Hosp. 2006;21:699703.
5. Vázquez C, Alcaraz F, Balsa JA, et al. Prevalence of psychiatric cases in overweight or obese patients attended in a hospital outpatient clinic]. Med Clin (Barc). 2008;130:41-46.

6. Willett WC, Dietz WH, Colditz GA. Guidelines for healthy weight. $N$ Engl J Med. 1999;341:427-434.

7. Hruby A, Manson JE, Qi L, et al. Determinants and Consequences of Obesity. Am J Public Health. 2016;106:1656-1662.

8. Buono G, Crispo A, Giuliano M, et al. Combined effect of obesity and diabetes on early breast cancer outcome:a prospective observational study. Oncotarget. 2017;8:115709-115817.

9. Walter S, Kunst A, Mackenbach J, et al. Mortality and disability:the effect of overweight and obesity. Int J Obes (Lond). 2009;33:1410-8.

10. da Costa Pimenta W, Santos Brant Rocha J, Prates Caldeira A, et al. Abdominal obesity and association with sociodemographic, behavioral and clinical data in climacteric women assisted in primary care. PLoS One. 2020;15(8): 0237336

11. El Khoudary SR, Greendale G, Crawford SL, et al. The menopause transition and women's health at midlife:a progress report from the Study of Women's Health Across the Nation (SWAN). Menopause. 2019;26:1213-27.

12. Greendale GA, Sternfeld B, Huang M, et al. Changes in body composition and weight during the menopause transition. JCI Insight. 2019;4.

13. Clinical Guidelines on the Identification, Evaluation, and Treatment of Overweight and Obesity in Adults-The Evidence Report. National Institutes of Health. Obes Res. 1998;6(Suppl 2):51S-209S.

14. Lizcano F, Guzmán G. Estrogen Deficiency and the Origin of Obesity during Menopause. Biomed Res Int. 2014;2014:757461.

15. Coyoy A, Guerra-Araiza C, Camacho-Arroyo I. Metabolism Regulation by Estrogens and Their Receptors in the Central Nervous System Before and After Menopause. Horm Metab Res. 2016;48(8):489-496.

16. Sanchez A, Labeira P, Gutierrez P, et al. Utility of indirect Calorimetry in the measure of the Resting energy expenditure (REE) in patiens with overweght or Obesity (PwO). European and International Congress of Obesity. EOICO. 2020.

17. Clotilde Vázquez, Alba Galdón, Ana I de Cos, et al. Successful Evolution of a Patient with Obesity, Metabolic Syndrome and Severe Gastroesophageal Reflux after Probiotic Intake. Advances in Obesity, Weight Management \& Control. 2015;2(1):00009.

18. Baker JM, Al-Nakkash L, Herbst-Kralovetz MM. Estrogen-gut microbiome axis:Physiological and clinical implications. Maturitas. 2017;103:45-53.

19. Clotilde Vázquez, Paula Labeira, Álvaro Sánchez, Resting energy Expenditure and Lipid Oxidation measured by Indirect Calorimetry and its relationship with clinical, lifestyle and body composition in post menopausal women. 62th Congress of the Sociedad Española de Endocrinologia y Nutricion ( SEEN ) Sevilla; 2021.

20. Chopra S, Sharma KA, Ranjan P, et al. Weight Management Module for Perimenopausal Women:A Practical Guide for Gynecologists. $J$ Midlife Health. 2019;10:165-172. 